\title{
Some Radiation and Photochemical Studies in Aqueous Polysaccharide Gel Systems*
}

\author{
D. J. Wedlock, ${ }^{* 1}$ G. O. Phillips, ${ }^{* 1}$ and J. K. Thomas ${ }^{* 2}$ \\ ${ }^{* 1}$ School of Natural Sciences, North E Wales Institute of \\ H.E. Connah's Quay, Clwyd, U.K. \\ ${ }^{*}$ Chemistry Department and Radiation Laboratory, University of Notre Dame, \\ Notre Dame, Indiana, 46556, U.S.A.
}

(Received December 26, 1978)

\begin{abstract}
The radiation and photochemistry of aqueous polysaccharide gel matrices have been investigated with a view to prolonging the lifetime of transient species, prior to storage of photochemical events. Our results indicate that with gels of kappa carrageenan and agarose, the rigid macroscopic gel structure is not reflected in the microscopic structure and reactions resemble closely those that would take place in aqueous solution under comparable conditions. This shows that the interior of the gel structure is essentially very fluid. Fluorescence probes were used in agarose gel systems incorporating neutral and anionic surfactants. Under such circumstances, there is strong evidence showing that a micellar-type environment exists, and also a gel-surfactant complex is formed with both neutral and anionic surfactants. The gel-surfactant complex appears on the basis of fluorescence quenching and fluorescence polarization studies, to have a more mobile interior than the interior of an ordinary surfactant micelle in the absence of the agarose. Studies on the stoichiometry of the complex indicate a much lower critical micelle concentration for the surfactant sodium dodecyl sulphate (SDS) in the presence of agarose. The agarose gel structure does not aid the micellar catalysed charge separation that occurs on photolysis of pyrene.

KEY WORDS Polyelectrolyte / Agarose / Sodium Dodecylsulphate / Pyrene Photoionization / Pulse Radiolysis / Fluorescent Probe /
\end{abstract}

The rate constants of several diffusion-controlled reactions of the solvated electron with solutes in viscous solvents such as glycerol were studied ${ }^{1.2}$ and are shown to be dependent on the reciprocal viscosity of the solutions, using systems such as glycerol-water and sucrose-water. Polyelectrolytes generally have considerable influence on the rheology of their solutions as well as on the diffusion properties of counterions and co-ions ${ }^{3-5}$ and the Donnan distribution of counterions and co-ions ${ }^{6}$ at relatively low concentrations of polyanion monomer. It might thus be expected that a polyanionic gel would influence the fate of reactive species such as the hydrated electron with sloutes by influencing diffusion properties and Donnan distribution of such

* The Radiation Laboratory of the University of Notre Dame is operated under contract with the U. S. Energy Research and Development Administration. species, in the light of recent findings. ${ }^{7}$

Electron transfer from one species to another is an early event in nearly every scheme for photochemical solar energy conversion, and as a precursor to photochemical energy storage, extending lifetimes and increasing the yields of reactive species such as radical cations and hydrated electron pairs is of obvious importance. ${ }^{8-11}$ The quantum yield of such processes is usually very low due to geminate recombination of primary species. In heterogeneous systems, the geminate recombination may be slowed effectively by anionic micellar species. In aqueous anionic micellar solutions of pyrene, hydrated electrons are ejected from pyrene in the hydrophobic micellar core in high quantum yield. ${ }^{12}$ In methanolic solution, the yield is low due to geminate recombination of the pyrene cation, $\mathrm{P}^{+}$, and the solvated electron. The solvated electrons that survive are short-lived compared to the micellar solution since they recombine with the pyrene cation in a 
diffusion-controlled process. In the micellar solution, the hydrated electron is prevented electrostatically from rapid recombination with the cation left in an anionic micelle.

It has been shown that anionic surfactant complexes between sodium dodecylsulphate (SDS) with charged and uncharged cellulose ethers exist, both above and below the critical micelle concentration (CMC) ${ }^{13}$ If such complexes exist not only for soluble and hence essentially mobile polysaccharide molecules, but also for waterinsoluble polysaccharides in the gelled state, one might expect such surfactant-gel complexes to exhibit behaviour which is different in character from that observed in normal aqueous micellar solution, in terms of influencing the lifetimes of transient species such as $\mathrm{P}^{+}$, because of restricted diffusion in the gel matrix.

To this end we have investigated certain radiation and photochemical processes in aqueous polysaccharide polyanion solutions, in aqueous poly-saccharide polyanionic gels and aqueous neutral polysaccharide gel/micellar systems to see what modifying effect a gel structure has on the rates of such events and to see if the infinitely high viscosity implied by lack of bulk flow of a gel, could provide additional enhancement of lifetimes, even perhaps to the extent of trapping species in gel compartments.

\section{EXPERIMENTAL}

\section{Materials}

Purified $\kappa$-carrageenan and agarose (electrophoretic grade) were kindly supplied by Marine Colloid Inc. Rockland, Maine, and were used without further purification. Pyrene from Eastman-Kodak was recrystallized from EtOH. Water was first distilled from a Barnsted still and subsequently from alkaline permangamate. Sodium dodecyl sulphate (SDS) was BDH specially pure type and Igepal CO-630 was obtained from industrial sources. All other reagents were of analytical grade.

\section{Sample Preparation}

$\kappa$-Carrageenan gels were prepared by dispersing the carrageenan on the surface of magnetically stirred water, with heating, the tared beaker being sealed with aluminium foil. The potassium chloride required for gelation was added from a $10 \% \mathrm{w} / \mathrm{v}$ aqueous solution when the carrageenan was completely dissolved to give a final concentration of $1 \% \mathrm{KCl}$ and $1 \% \kappa$-carrageenan. Water lost by evaporation was replaced. The $\kappa$-carrageenan- $\mathrm{KCl}$ mixture was thoroughly agitated to ensure complete mixing at about $60^{\circ} \mathrm{C}$. When $\mathrm{NO}_{3}{ }^{-}$was added, it was introduced as $\mathrm{KNO}_{3}$ with the $\mathrm{KCl}$, from a concentrated solution, to give the desired final concentration. The mixture was kept in sol form with heating, on a hot plate, whilst degassing with nitrogen was carried out for pulse radiolysis experiments. When $\cdot \mathrm{OH}$ radical reaction rates were determined by pulse radiolysis the sol form was flushed with $\mathrm{N}_{2} \mathrm{O}$ maintaining the system just above its gelling temperature to ensure maximum solubility of $\mathrm{N}_{2} \mathrm{O}$. Molarities were based on the disaccharide unit.

Agarose gels were prepared either in an aqueous medium or in an aqueous surfactant medium by dispersing the agarose in water or a surfactant solution of the appropriate concentration. Again the vessel was sealed with aluminium foil and a magnetic stirrer used. The vigourously stirred solution was boiled for at least two minutes. Weight loss after boiling was made up by addition of water. Fluorescent probes were incorporated by injecting the appropriate amount from concentrated stock solutions in volatile organic solvents, straight into $1 \mathrm{~cm}$ fluorescence cells with ground glass tops. The volatile organic solvent was evaporated off with a nitrogen stream. The hot agarose sol was pipetted into the cell $(4 \mathrm{~m} l)$, stoppered and shaken vigourously to ensure thorough dissolution and incorporation of the probe. The agarose gel was maintained just above its gelling point $\left(35^{\circ} \mathrm{C}\right)$ and the flushing gas (usually $\mathrm{N}_{2}$ but $\mathrm{N}_{2} \mathrm{O}$ for $\cdot \mathrm{OH}$ radical adduct determinations) was introduced through a Pasteur pipette. For the fluorescence quenching studies, $\mathrm{Tl}^{+}$was introduced into the original water, $\mathrm{CH}_{3} \mathrm{NO}_{2}$ was introduced by injection to the agarose sol, after degassing, under a nitrogen atmosphere. Agarose sols with all components; in a degassed state, were sealed in the fluorescence cell, thoroughly mixed, given moderate heat to allow air bubbles to rise and then allowed to gel for 20 minutes. The gelation was always brought about by natural, not rapid cooling.

\section{Apparatus}

Static fluorescence was carried out using an 
Aminco-Bowman spectrofluorometer. Fluorescence polarization was determined using a Elscint fluorescence polarimeter. Laser photolysis was carried out on pyrene located in micelles excited with $10 \mathrm{~ns}$ pulses of a $347.1 \mathrm{~nm}$ line from a Q-switched, frequency-doubled, ruby laser. The excitation produces excited states of pyrene. The reaction of the short-lived intermediates produced was observed by fast kinetic emission or absorption spectrophotometry. ${ }^{14}$ Pulse radiolysis was caried out using $10 \mathrm{~s}$ pulses of $10 \mathrm{MeV}$ electrons from a linear accelerator. ${ }^{1} \cdot \mathrm{OH}$ radical reaction rates in the various polysaccharide systems were determined by competition kinetics using the $(\mathrm{CNS})_{2}^{-}$radical anion as a probe, in $\mathrm{N}_{2} \mathrm{O}$ saturated systems. ${ }^{15}$ Two concentrations of $\mathrm{CNS}^{-}$were used for each determination, 1 and $0.3 \mathrm{mM}$. The results are based on a rate constant of $6.6 \times 10^{9} \mathrm{~mol}^{-1} \mathrm{~s}^{-1}$ for the reaction of $\cdot \mathrm{OH}+\mathrm{CNS}^{-}$.

\section{RESULTS AND DISCUSSION}

\section{Pulse Radiolysis in Gel Systems}

Pulse-radiolysis experiments were carried out on $\kappa$-carrageenan gels and sols and on agarose gels. Values of $k_{2}$, the second-order rate constant, for decay of the hydrated electron in these media, were obtained under pseudo first-order conditions. Further, the influence of these media on the reaction of the hydrated electron with the nitrate anion was observed, in this diffusion-controlled reaction

$$
\mathrm{e}_{\mathrm{aq}}{ }^{-}+\mathrm{NO}_{3}{ }^{-} \rightarrow \mathrm{NO}_{3}{ }^{2-}
$$

The hydrated electron reacts slowly with $\kappa$-carrageenan both in the sol phase and in the gel phase (Table I). The nearly threefold increase in the reactivity of $\mathrm{e}_{\mathrm{aq}}{ }^{-}$in the $\kappa$-carrageenan gel compared to the $\kappa$-carrageenan sol, can be partially rationalized in terms of the ionic-strength effect according to the Brönsted-Debye equation ${ }^{16}$

$$
\log _{10} \frac{k}{k_{0}}=1.02 z_{\mathrm{a}} z_{\mathrm{b}} \frac{\mu}{1+a \mu^{1 / 2}}
$$

where $k$ is the rate constant at ionic strength $\mu, k_{0}$ is the rate constant at infinite dilution of ions and $a$ is an adjustable parameter depending on the distance of closest approach between A and B, expected to lie between 1 and 3 (we have assumed a value of 2), $z_{\mathrm{a}}$ and $z_{\mathrm{b}}$ being the charges carried by the reacting species. As the actual fate of the electron in pure $\kappa$ carrageenan sol and gel systems and in agarose gels is somewhat uncertain, explanations for variations in the electron lifetime are somewhat less important than the observation that no apparent trapping of electrons occurs in the gel matrix nor are any unusually long lifetimes of electrons observed.

$e_{\mathrm{aq}}{ }^{-}$Reaction with $\mathrm{NO}_{3}{ }^{-}$in Polysaccharide Networks

The influence of gel structure on the diffusioncontrolled reaction of the hydrated electron with $\mathrm{NO}_{3}{ }^{-}$is negligible. In the essentially neutral gel network of agarose, the value of $k_{2}$ for reaction (1) is identical with that in homogeneous aqueous solution, at $1.1 \times 10^{10} \mathrm{~s}^{-1} \mathrm{M}^{-1}$. The enhancement of the rate by $\sim 50 \%$ (Table I), observed in the polyanionic $\kappa$-carrageenan gel, could be explained entirely within experimental error in terms of the ionic-strength effect of the added $\mathrm{KCl}$, used to cause the $\kappa$-carrageenan to gel. According to eq 1 , the ratio of the rate constants for $\mathrm{e}_{\mathrm{aq}}{ }^{-}+\mathrm{NO}_{3}{ }^{-}$should be 1.6 (assuming $\mathrm{KCl}$ gives rise to the ionic strength). The ratio of the rate constants in practice is found to be 1.5 , using a value of $a=2$. Invoking Donnan effects ${ }^{7}$ to explain the difference in rate constants for reaction (1) in the agarose gel network and the $\kappa$ carrageenan- $\mathrm{KCl}$ gel network, which have essentially identical sugar backbones, i.e., 1,3-linked galactose and 1,4-linked 3,6-anhydrogalactose,

Table I. Rate constant at ionic strength for hydrated reactions

\begin{tabular}{cccc}
\hline & & $k / \mathrm{dm}^{-3} \mathrm{~mol}^{-1} \mathrm{~s}^{-1}$ & \\
\cline { 2 - 4 } Reactions & $\kappa$-Carrageenan (sol) & $\kappa$-Carrageenan (gel) & Agarose gel \\
\hline $\mathrm{e}_{\mathrm{aq}}^{-}+\mathrm{Polymer}_{\mathrm{aq}}^{-}+\mathrm{NO}_{3}^{-}$ & $2.16 \times 10^{7}$ & $6.05 \times 10^{7}$ & $4.70 \times 10^{7}$ \\
$\cdot \mathrm{OH}+$ Polymer & $1.08 \times 10^{10}$ & $1.61 \times 10^{10}$ & $1.10 \times 10^{10}$ \\
$1.46 \times 10^{8}$
\end{tabular}

a Diphenylhexatriene was the fluorescent probe. 
would be insufficient since Donnan effects in the $\kappa$ carrageenan polyanion gel network will be small or suppressed altogether by the addition of the $\mathrm{KCL}$ used to cause gelation.

\section{OH Adducts of Polysaccharide Sol and Gels}

The rates of reaction of $\cdot \mathrm{OH}$ radicals investigated by competion kinetics with both gel types are essentially the same, and thus the potential field of the $\kappa$-carrageenan gel has only little influence on the rate of formation of the $\cdot \mathrm{OH}$ adduct. Also the rate of formation of the $\cdot \mathrm{OH}$ adduct of the $\kappa$-carrageenan sols is only moderately greater than that of the $\kappa$ carrageenan gel. Immobility of the polyanion thus does not greatly effect the rate. The values of $k_{2}$ for . $\mathrm{OH}+\kappa$-carrageenan are close to those respectively for heparin ${ }^{17}$ and carboxymethylcellulose ${ }^{18}$ at 2.2 and $1.97 \times 10^{8} \mathrm{M}^{-1} \mathrm{~s}^{-1}$ indicating a near constancy of rate of formation of the $\cdot \mathrm{OH}$ adduct for all these anionic polysaccharides, irrespective of whether they are in a sol or gel form.

Pulse-radiolysis studies of these polysaccnaride gel systems tends to indicate that the gel matrix does not alter the viscosity of the water component of the gel on a microscopic level. The system is essentially a very fluid one, and such behaviour as was observed in aqueous glycerol solutions ${ }^{1,2}$ where the rate constants of $\mathrm{e}_{\mathrm{aq}}{ }^{-}$reactions demonstrated a dependence on the inverse square root of the bulk viscosity, are absent in these polysaccharide gel systems. In the polyanionic gel of $\kappa$-carrageenan, there is no evidence to suggest that trapping of $\mathrm{e}_{\mathrm{aq}}$ occurs in compartments formed by the negative electrostatic potential of the sulphate ester groups.

\section{Pyrene in Micellar and Micellar-Gel Environments}

Steady state fluorescence spectra of pyrene

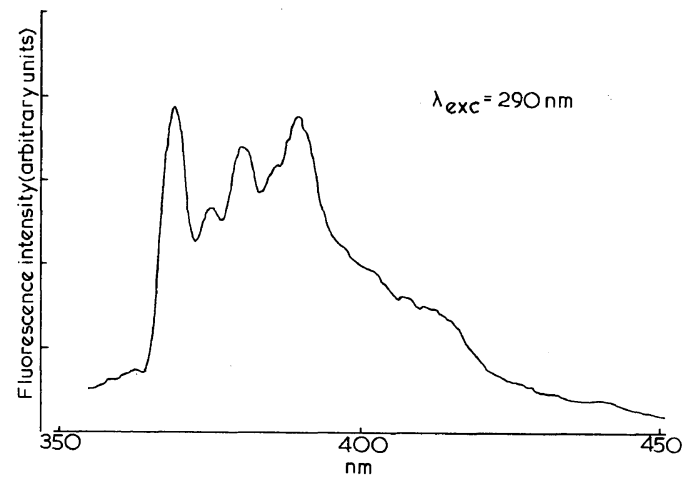

Figure 1. Fluorescence spectra of pyrene $(10 \mu \mathrm{M})$ incorporated in an SDS $\left(4 \times 10^{-2} \mathrm{M}\right)$-agarose $(2 \%)$ matrix.

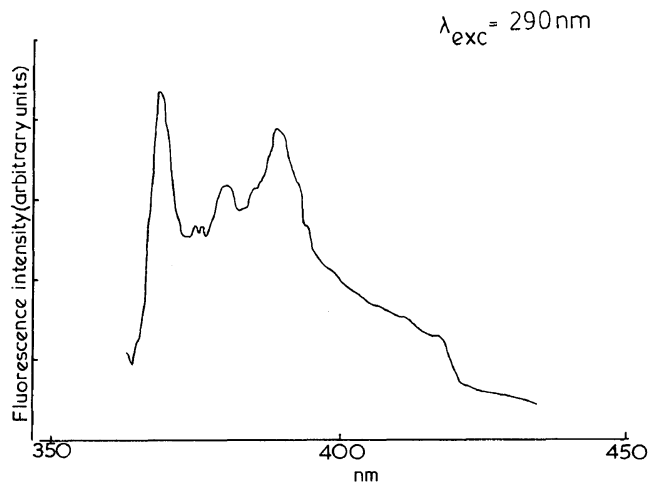

Figure 2. Fluorescence spectra of pyrene $(10 \mu \mathrm{M})$ incorporated in an Igepal $\left(1 \times 10^{-2} \mathrm{M}\right)$-agarose $(2 \%)$ matrix.

incorporated in SDS and Igepal CO-630 micelles in aqueous solutions containing $2 \% \mathrm{w} / \mathrm{v}$ to give agarose gel-surfactant systems, are shown in Figures 1 ane 2. The measured III/I ratio of the vibronic fine structure in both systems can be used to indicate the

Table II. Fluorescence data for pyrene $(10 \mu \mathrm{M})$ solubilized in various surfactant systems

\begin{tabular}{lcccc}
\hline & NaLS aq & NaLS agarose & Igepal CO-630 aq & $\begin{array}{c}\text { Igepal CO-630 } \\
\text { agarose }^{\mathrm{a}}\end{array}$ \\
\hline III/I & 0.88 & 0.87 & 0.77 & 0.78 \\
Excimer/Monomer & - & - & $0.42^{\mathrm{b}}$ & $0.56^{\mathrm{b}}$ \\
$P^{\mathrm{c}}$ & 0.105 & 0.061 & 0.187 & 0.127 \\
$F$ & $320 \mathrm{~ns}$ & $320 \mathrm{~ns}$ & $263 \mathrm{~ns}$ & $287 \mathrm{~ns}$ \\
\hline
\end{tabular}

a Agarose concentration $2 \% \mathrm{w} / \mathrm{v}$.

b Pyrene concentration $3 \times 10^{-4} \mathrm{M}$.

c Diphenylhexatriene was the fluorescent probe. 
environment of the pyrene probe molecule. ${ }^{19}$ The pyrene spectra in the gel-surfactant systems indicate for both SDS $\left(4 \times 10^{-2} \mathrm{M}\right)$ and Igepal CO-630 $\left(1 \times 10^{-2} \mathrm{M}\right)$ when incorporated in $2 \%$ agarose gels, at concentrations above their normal critical micelle concentration (CMC), that pyrene is incorporated in a micellar-type environment, the values of the III/I ratio being almost identical to the literature values in normal aqueous micellar solutions. ${ }^{19}$ The excitation wavelength used for pyrene was lower at $275 \mathrm{~nm}$ than the more usual $330 \mathrm{~nm}$ used, in order to minimize scattering of the excitation beam by the gel structure, in the observed emission range. In the Igepal CO-630 surfactant system, at concentrations of pyrene $\left(10^{-4} \mathrm{M}\right)$ in Igepal CO-630 $\left(1 \times 10^{-2} \mathrm{M}\right)$, an emission band centred around $470 \mathrm{~nm}$ is observed in both aqueous solutions of Igepal CO-630-pyrene and in Igepal CO-630-pyrene-agarose gel. This emission is ascribed to the excimer emission of pyrene ${ }^{20.21}$ (such excimer formation is not observed for pyrene in SDS micelles). When allowance is made for the underlying scatter due to the gel network by using a gel sample of the same agarose concentration, as a blank, the values of the excimer/monomer peak ratios (Table II), indicate more extensive excimer formation in the surfactant-gel system than in aqueous micellar solution. Such observations are consistent with a more fluid interior for the hydrophobic part of the surfactant aggregates in the gel system, compared to normal aqueous solution since pyrene-pyrene encounters leading to excimer formation will be more frequent in a more fluid interior.

\section{Fluorescence Polarization Studies}

The degree of polarization of the emitted fluorescence from a suitable fluorescent probe can serve as a measure of the local or microviscosity of the probe surroundings. ${ }^{22}$ The relationship between the degree of polarization and the microviscosity is given by Perrins equation,

$$
\left(\frac{1}{P}-\frac{1}{3}\right)\left(\frac{1}{P_{0}}-\frac{1}{3}\right)=1+\frac{k T \tau_{\mathrm{F}}}{\eta V_{0}}
$$

where $p_{0}$ is the fluorescence polarization in a slovent of extremely high viscosity; $\tau_{\mathrm{F}}$ is the lifetime of the fluorescent excited state; $T$ is the temperature in $\mathrm{K} ; k$ is Boltzmann's constant; $v_{0}$ is the effective volume of the fluorescent molecule. The probe 1,6-diphenyl- hexatriene (DPHT) is used to compare the fluidity of the micellar core for micelles formed by Igepal CO630 and SDS, in aqueous solution and the species present in an agarose gel-surfactant maxtrix. The theoretical limit of $P$ is 0.45 at the applied excitationwavelength. Comparison of the degree of polarization $P$, of the DPHT emission (Table II), shows that for both surfactants, SDS and Igepal CO630, $P$ takes substantially lower values when the surfactant is incorporated in the gel matrix than when it is in simple aqueous solution. The depolarization of the emitted light can occur either by movement of the probe within the micelle or rotation of the micelle as a whole. As the latter process is reasonably unlikely to be enhanced by a gel network, it is the former process that is thought to be enhanced by it. Since $\tau_{\mathrm{F}}$, the fluorescent lifetimes measured for pyrene (Table II), are shown to be fairly constant for both SDS and Igepal CO-630 in micellar solutions of surfactant and in the agarose-surfactant system, it is reasonable that $\tau_{\mathrm{F}}$ of DPHT should remain the same in the two systems. Therefore, the observed decrease in $P$ is attributed to an increase in the degree of fluidity and hence in the freedom of movement of the DPHT probe. This can be interpreted in terms of an expansion of SDS and Igepal CO-630 micellar-type species by incorporation of a proportion of the agarose in the micelle or more specifically adsorption of the surfactant on to the gel network at hydrophobic sites. It should be remembered that agarose îs coldwater-insoluble and hence forms a gel structure, particularly because of the hydrophobic nature of the 3,6-anhydrogalactose sugar unit. If adsorption of micellar-like clusters on the gel network occurred, then it would be likely that the hydrophobic core of the micelle would attach itself to this sugar unit, or rearrange itself so as to include it in the core. This would cause an expansion of the micelle and hence a greater fluidity in the core, in a similar manner to the mixed micellar systems ${ }^{23,24}$ observed for longalkylchain alcohols. Further evidence that these observations are reasonable is that poly(ethylene oxide)-SDS aggregates have been observed and that these aggregates can be described as a mixed micellar system. $^{25.26}$

\section{N-Phenyl-1-Naphthylamine}

The fluorescent probe $N$-phenyl-1-naphthylamine (NPN) has been used ${ }^{27}$ as a means of indicating the 


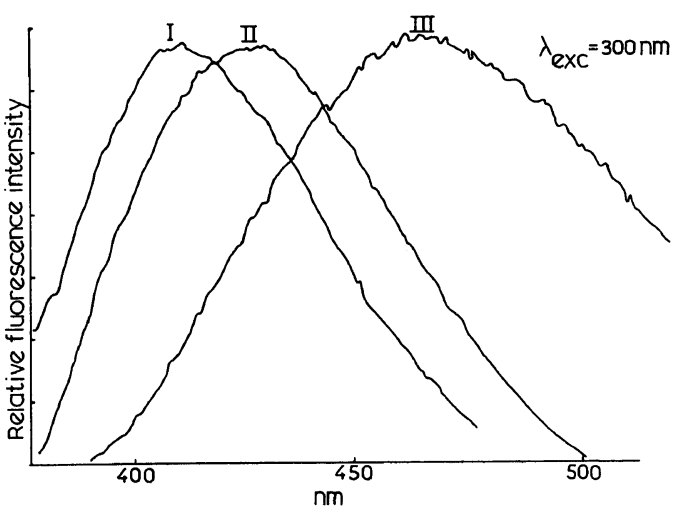

Figure 3. $N$-phenyl-naphthylamine $(50 \mu \mathrm{M}$ in I and II, $100 \mu \mathrm{M}$ in III) incorporated in: I, SDS $(4 \times$ $\left.10^{-2} \mathrm{M}\right)$-agarose $(2 \%)$; II, SDS $\left(4 \times 10^{-2} \mathrm{M}\right)$ micelles; III, $\mathrm{H}_{2} \mathrm{O}$.

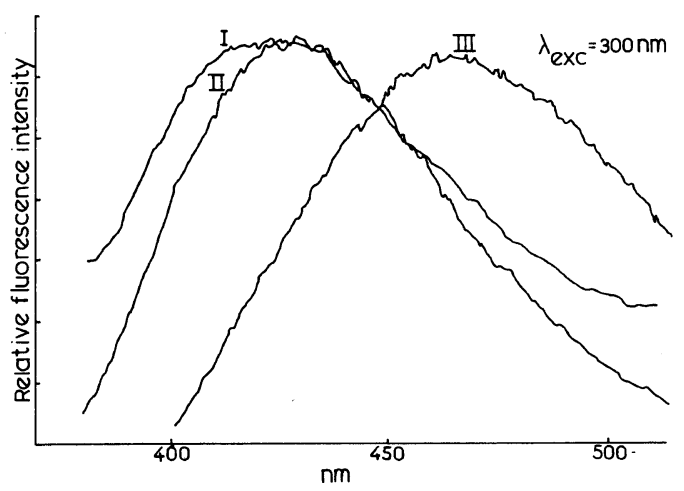

Figure 4. $N$-phenyl-1-naphthylamine $(50 \mu \mathrm{M}$ in $\mathrm{I}$ and II, $100 \mu \mathrm{M}$ in III) incorporation in: I, Igepal CO-630 $\left(1 \times 10^{-2} \mathrm{M}\right)$-agarose $(2 \%) ;$ III, Igepal $\left(1 \times 10^{-2} \mathrm{M}\right)$ micelles; III, $\mathrm{H}_{2} \mathrm{O}$.

polarity of the environment in which it resides, particularly in cell membranes. It is thought by some workers to reside in the hydrocarbon core ${ }^{28}$ and by others to lie at the interface of the polar-nonpolar region $^{27}$. In our application, spectrofluorimetric measurements were made (Figures 3 and 4 ) of NPN in water, in aqueous solutions of SDS and Igepal CO-630 micelles, in $2 \%$ agarose gel-SDS and $2 \%$ agarose gel-Igapal CO-630 micellar systems, for each surfactant used, and all three spectra of NPN fluorescence are shown. A common feature for both surfactants is that a pronounced blue shift is seen in the $\lambda_{\max }$ of the NPN as its environment is changed from aqueous solution to aqueous micellar solution to aqueous gel-surfactant solution. This is indicative of a more hydrophobic environment for the probe in the agarose gel-surfactant system than in the simplemicellar-solution environment for both surfactants studied. Thus we have further indication that we are looking at a micellar-type species or a surfactant aggregate in the gel system, and that as well as being more fluid, appears more hydrophobic. Fluorescence spectra of NPN incorporated in SDS-agarose gel systems were obtained for different concentrations of SDS. A discontinuity was found in the plot of relative fluorescence intensity $v s$. concentration of SDS (Figure 5). We ascribe this discontinuity at $3 \times 10^{-3} \mathrm{M}$ SDS to the onset of surfactant cluster or aggregate formation in this system. This is lower than the literature value ${ }^{19}$ for the normal CMC of SDS and this lowering of CMC is typical of behaviour observed in polymer surfactant solutions. ${ }^{29}$ It should be noted that there are other causes of lowering of CMC's such as an increase in the ionic strength on addition of an electrolyte, although no ionic strength increase of any significance is expected on addition of $2 \%$ agarose as it is essentially devoid of ester sulphate. In dye solubilization studies for $(0.1 \%)$ methylcellulose-SDS mixtures the onset of ${ }^{29}$ SDS binding to methylcellulose first occurred at a concentration of $4 \times 10^{-3} \mathrm{M} \mathrm{SDS}$, and it was thought of in terms of a slight residual positive charge on its ether oxygens, initiating a process of cluster formation. It would thus seem highly likely that it is surfactant clusters attached to the agarose gel network that are formed at the critical concentration of $3 \times 10^{-3} \mathrm{M}$.

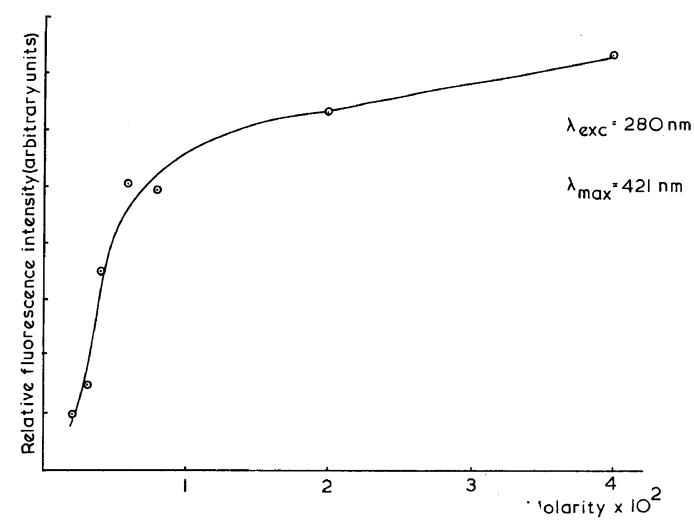

Figure 5. Plot of change in relative fluorescence intensity of $N$-phenyl-1-naphthylamine $(50 \mu \mathrm{M})$ solubilized in varying concentrations of SDS in a $2 \%$ agarose matrix. 
Furthermore, equilibrium-dialysis measurements in poly( $N$-vinylpyrolidone)-SDS systems ${ }^{30.31}$ indicate the onset of low-order clusters or complexes, prior to true mixed-micelle formation which occurs at a concentration of free SDS lower than the regular CMC. It was noted in these studies that the concentration of free SDS required for the formation of SDS clusters, decreased with increasing polymer concentration. On comparing the concentration of agarose $(2 \% \mathrm{w} / \mathrm{v})$ used for creating the agarose gel network with the concentration of methylcellulose $(0.1 \%)$ used in the dye-solubilization studies, it is likely that the concentration for a cluster-type complex formation has been reached.

\section{Fluorescence Quenching Studies}

Pyrene fluorescence emission can also give information on the state of surfactant micelles incorporated in normal aqueous solution and in the aqueous agarose gel system as it normally has a sufficiently long lifetime $(>100 \mathrm{~ns})$, in the excited singlet state, so that substantial diffusion can occur before emission. Change in the micellar structure will affect diffusion of pyrene and hence encounters with quenchers of its excited singlet state. Some correlation should thus be obtained between quenching-rate constants and the state of micellar entities sloubilizing pyrene.

The molecule nitromethane and the thallous ion quench the excited singlet state of pyrene,$^{32} \mathrm{P} *$. With quencher concentrations much greater than that of the excited pyrene molecule, the decay of the pyrene fluorescence is pseudo first order, approximating to an exponential form. The quenching-rate constant can be calculated from eq 4

$$
k_{1}=k_{0}+k_{2}[\mathrm{Q}]
$$

where $k_{1}$ and $k_{0}$ are the rate constants for decay of $\mathrm{P}^{*}$ in the presence and absence of quencher, $\mathrm{Q}$, and $k_{2}$ is the quenching-rate constant. The concentration of pyrene is sufficiently low so that no pyrene excimer formation occurs. It can be seen that the lifetime of $\mathrm{P}^{*}$ is not affected by the agarose gel network, when solubilized in either SDS or Igepal CO-630 surfactant systems (Table II). When nitromethane is used as a quencher of pyrene fluorescence in both Igepal CO-630 and SDS, aqueous micellar solution and in the agarose gel-surfactant systems, $k_{2}$ is greater in the gel-surfactant system than in ordinary aqueous micellar solution for both surfactants. This again is indicative of a more mobile hydrophobic interior when the surfactant is associated with the agarose gel network. The values of $k_{2}$ when the thallous ion is used as a quencher, are virtually identical for Igepal CO-630 in aqueous micellar solution and in the agarose gel-surfactant system. This is reasonable as the thallous ion is not particularly hydrophobic and would hence not closely approach this neutral micelle and not appreciate the expanded, more fluid, hydrophobic interior. In the case of SDS with $\mathrm{T}^{+}{ }^{+}$as quencher, $k_{2}$ is again greater in the agarose gel-surfactant system than in the aqueous micellar system, indicative now of both the surface of the cluster, as well as the core being in a more fluid state when associated with the gel. The approach of the thallous cation to the SDS micellar surface is obviously significantly greater than its approach to Igepal CO-630, because of the surface charge of the head groups, explaining the relative difference in $k_{2}$ values for Igepal CO-630 and SDS. Because of the more intimate contact of the thallous ion with the SDS micelle, the more fluid nature of the SDS cluster in the agarose gel system gives rise to a greater $k_{2}$ value in the gel-surfactant system compared to the aqueous micellar solution.

\section{Photoionization of Pyrene}

When pyrene is solubilized in an anionic micelle

Table III. Rate constants $\left(10^{9} \mathrm{M}^{-1} \mathrm{~s}^{-1}\right)$ for quenching reactions

\begin{tabular}{|c|c|c|c|c|}
\hline Reaction & $\operatorname{SDS}^{\mathrm{a}}$ & SDS/Agarose ${ }^{\mathbf{b}}$ & Igepal CO- $630^{\mathrm{a}}$ & $\begin{array}{c}\text { Igepal } \\
\text { CO-630/ } \\
\text { Agarose }^{b}\end{array}$ \\
\hline $\begin{array}{l}\mathrm{Py}^{*}+\mathrm{CH}_{3} \mathrm{NO}_{2} \\
\mathrm{Py}^{*}+\mathrm{Tl}^{+}\end{array}$ & $\begin{array}{c}3.15 \\
\tau<3 \times 10^{-8} \mathrm{~s}\end{array}$ & $\begin{array}{c}10.10 \\
\tau<2 \times 10^{-8} \mathrm{~s}\end{array}$ & $\begin{array}{l}0.55 \\
0.12\end{array}$ & $\begin{array}{l}0.77 \\
0.15\end{array}$ \\
\hline
\end{tabular}

a $\operatorname{SDS} 4 \times 10^{-2} \mathrm{M}$ Igepal CO-630 $1 \times 10^{-2} \mathrm{M}$.

b Agarose $2 \% \mathrm{w} / \mathrm{v}$. 
such as SDS, its two photon photoionization according to eq 5

$$
\mathrm{P}_{\text {micellar }} \underset{341 \mathrm{~nm}}{\stackrel{2 h v}{\longrightarrow}} \mathrm{P}_{\text {micellar }}^{+}+\mathrm{e}_{\mathrm{aq}}^{-}
$$

is catalyzed, due to efficient escape of the electron from the micelle and subsequent maintenance of charge separation by repulsive forces from the micellar head groups. ${ }^{33}$ The fate of the hydrated electron formed by photoionization was monitored when the micellar-type species of SDS was formed in an agarose gel environment and in normal aqueous micellar solution. The ratio of the first-order rate constants for the decay of $\mathrm{e}_{\mathrm{aq}}^{-}$formed by photoionization in these systems, is given in Table IV. It has been noted that increasing micellar size ${ }^{33}$

Table IV. Photoionization of pyrene in SDS and SDA-agarose systems $\mathrm{s}^{\mathbf{a}}$

\begin{tabular}{cc}
\hline Reaction & $\frac{\text { SDS-agarose }}{\mathrm{SDS}}$, rate ratio \\
\hline $\mathrm{e}_{\mathrm{aq}}$ decay $\left(k_{1}\right)$ & 1.162 \\
$\mathrm{P}^{+}$decay $\left(k_{2}\right)$ & 0.835 \\
\hline
\end{tabular}

a Pyrene $10 \mu \mathrm{M}$, SDS $4 \times 10^{-2} \mathrm{M}$, agarose $2 \%$.

leads to a decrease in the yields of photoionization due to failure of the electron to escape from the larger micelles. In bilayers such as lecithin, the large phosphocoline head groups greatly reduce the escape of $\mathrm{e}_{\mathrm{aq}}^{-}$to the aqueous phase, and similarly the great difficulty of the return of $\mathrm{e}_{\mathrm{aq}}^{-}$from the water phase to the pyrene cation is reflected in enhanced $\mathrm{e}_{\mathrm{aq}}^{-}$lifetimes. The virtually identical rates of $\mathrm{e}_{\mathrm{aq}}^{-}$decay in the surfactant-gel system and the normal aqueous micellar solution infer that there is no polymer influence on the surfactant (either free or adsorbed) micellar clusters which endow it with bilayer-like properties. Also the gel structure does not seem to reduce micellar-solubilized-pyrene radical cationhydrated-electron interactions. The radical cation of pyrene, formed in SDS micelles has a half life of about $20 \mathrm{~ms}$ in normal aqueous micellar solution ${ }^{33}$ and the ratio of the second-order rate constans of decay of $\mathrm{P}^{+}$in the surfactant-gel system compared to normal aqueous micellar solution is $0.835 / 1$, showing a slight, but not significant enhancement of $\mathrm{P}^{+}$lifetime in the gel environment (Table IV).

\section{CONCLUSIONS}

Radiolytic and photochemical studies show that the basic kinetics of diffusion-controlled reactions are little affected by carrageenan and agarose gels. Although the macrostructure of these systems is severely restricted compared to dilute aqueous solutions, nevertheless the movement of small species on a microscopic scale is unhindered. The network structure of the gel provides a template which facilitates the micellar-aggregate formation, on addition of surfactants to these systems. The resultant aggregates are less rigid than the unadulterated micelle structure. Basic differences in kinetic and spectroscopic patterns of the gel-micelle structures are noted along with a change in the CMC of the surfactant. With NPN as a fluorescent probe, the $\lambda_{\max }$ is dependent on the presence of a polysaccharide network when surfactants are present as aggregates. This is also reflected in the polarization of the fluorescence of DPHT. When Igepal CO-630-agarose gel mixtures are investigated, it is seen that excimer/monomer ratios of pyrene are dependent on the presence of a polysaccharide network. Similarly, fluorescencequenching studies indicate perturbation of surfactant aggregates by the gel.

The photochemicl species formed in laser-flash photolysis, such as $\mathrm{e}_{\mathrm{aq}}^{-}$and arene cations are not significantly affected by the gel structure in terms of decay rates. This indicates a rather open network for the gel. It is hoped that other gel-micelle systems can be constructed that will isolate reactions to a greater extent than those studied here.

\section{REFERENCES}

1. T. Kajiwara and J. K. Thomas, J. Phys. Chem., 76, 1700 (1972).

2. B. Cercek, Int. J. Radiat. Phys. Chem., 7, '23 (1975).

3. H. Magdelenat, P. Turq, and M. Chemla, Biopolymers, 13, 1535 (1974).

4. H. Magdelenat, P. Turq, and M. Chemla, Biopolymers, 15, 175 (1976).

5. A. Kowblansky, R. Sasso, V. Spagnuola, and P. Ander, Macromolecules, 10, 78 (1977).

6. G. Manning, J. Chem. Phys., 51, 934 (1969).

7. J. E. Scott, V. Davies, and M. Ebert, Biochem. Soc. Trans., 3, 436 (1975).

8. S. A. Alkaitis, G. Beck, and M. Grätzel, J. Am. Chem. Soc., 97, 5723 (1975).

9. S. A. Alkaitis, M. Grätzel, and A. Henglein, Ber. 
Bunsenges Phys. Chem., 79, 541 (1975).

10. M. Grätzel and J. K. Thomas, J. Phys. Chem., 78, 2248 (1974)

11. M. Grätzel, A. Henglein, and E. Janata, Ber Bunsenges Phys. Chem., 79, 475 (1975).

12. P. Picuilo and J. K. Thomas, J. Chem. Phys. 68, 3260 (1978).

13. E. D. Goddard and R. B. Hannan "Micellization, Solubilization and Microemulsions", Vol.2, K. L. Mittal, Ed., Plenum Press, New York, N. Y., 1977.

14. J. K. Thomas, K. Johnson, T. Klippert, and R. Lowers, J. Chem. Phys., 48, 1608 (1968).

15. (a) G. E. Adams and J. W. Boag, J. H. Baxendale, M. Ebert, J. P. Keene, and A. J. Swallow, Ed., "Pulse Radiolysis", Academic Press, New York, N. Y., 1965, p 171; (b) J. H. Baxendale, P. L. T. Bevan, and D. A. Stott, Trans. Faraday Soc., 68, 2389 (1968); (c) J. H. Baxendale and D. A. Stott, Chem. Commun., 669 (1967).

16. A. J. Swallow, "Radiation Chemistry", Longman, London, 1973, p 140.

17. J. S. Moore, G. O. Phillips, J. V. Davies, and K. S. Dodgson, Carbohydr. Res., 12, 253 (1970).

18. O. I. Micic, B. H. Milosavljevic, G. O. Phillips, and D. J. Wedlock, J. Chem. Soc. Faraday Trans. 2, in press.

19. K. Kalyanasundaram and J. K. Thomas, J. Am. Chem. Soc., 99, 2039, (1977).

20. H. E. Edwards and J. K. Thomas, Carb. Res., 66, 333 (1978).
21. K. Kalyanasundaram, M. Grätzel, and J. K. Thomas, J. Am. Chem. Soc., 97, 3915 (1975).

22. M. Wong, J. K. Thomas, and M. Grätzel, J. Am. Chem. Soc., 98, 2391 (1976).

23. K. Shinoda in "Colloidal Surfactants", E. Hutchinson, Ed., Academic Press, New York, N. Y., 1963.

24. M. J. Schick, J. Am. Chem. Soc., 43, 681, (1966).

25. B. Cabane, J. Phys. Chem., 81, 1639 (1977).

26. K. Shirahama and N. Ide, J. Colloid Interface Sci., 54, 450 (1976).

27. H. E. Edwards, J. K. Thomas, C. F. Kulpa and G. Burleson, Biochim. Biophys. Acta, 448, 451 (1976).

28. G. K. Radda in "Biological Membranes", D. S. Parsons, Ed., Clarendon Press, Oxon. U. K., 1975, p 87.

29. E. D. Goddard and R. B. Hannan, "Micellization, Solubilization and Microemulsions", Vol. 2, K. L. Mittal, Ed., Plenum Press, New York, N. Y., 1977.

30. M. L. Fishman and F. R. Eirich, J. Phys. Chem., 75, 3135 (1971).

31. M. L. Fishman and F. R. Eirich, J. Phys. Chem., 79, 2740 (1975).

32. S. Cheng, M. Grätzel, J. K. Thomas and C. F. Kulpa, "Fast Processes in Radiation Chemistry and Biology", G. E. Adams and B. D. Micheal, Ed., John Wiley, London, 1975.

33. S. C. Wallace, M. Grätzel, and J. K. Thomas, Chem. Phys. Lett., 23, 359 (1973). 